\title{
Errors Introduced by Finite Space and Time Increments in Dynamic Response Computation
}

\author{
Samuel Levy and Wilhelmina D. Kroll
}

\begin{abstract}
An investigation is made of the accuracy and stability of numerical integration methods when applied to the computation of the dynamic response of structures to impact loads. The effect of finite time increments is studied both by obtaining analytical solutions for a single-degree-of-freedom system and by carrying out numerical integrations for manydegree-of-freedom systems; the effect of finite space increments is studied by replacing a continuous beam by a discrete number of elastically connected point masses. It is found that: (1) Of the methods investigated, only Houbolt's is stable when the time increments are large compared with the natural periods of the system. Errors are introduced by Houbolt's method, in this case, which result in the damping out of the responses in the higher modes of vibration. All of the methods give good results when the time increment is less than about $1 / 30$ of the period in the highest frequency mode. (2) The distributed mass of a beam can be considered to be concentrated at relatively few mass points for computational purposes; using a five mass idealization, the bending moment at the center of a uniform beam is determined with good accuracy.
\end{abstract}

\section{Introduction}

With larger aircraft, higher landing speeds, and the necessity of flying in bad weather, the transient vibrations caused by severe gusts, landing impacts, and similar shock loads are becoming increasingly important in the stress analysis of airplanes. The usual method of computing these transients is to superpose the response in a small number of the important modes $\left[\begin{array}{ll}1, & 2\end{array}\right] .{ }^{1}$ Such computations are lengthy and in some cases give results that are of questionable accuracy [3].

Houbolt [4] presents a numerical integration method in which the derivatives in the equations of motion are replaced by finite differences to permit a step-by-step calculation of the dynamic response of an elastic aircraft entering a gust. Houbolt's method is adaptable to the problem of the dynamic response of an airplane to landing impact and was used [5] to determine the deflections of the wing for an unsymmetrical two-point landing of a model airplane. From these deflections, the bending moments on each wing at stations 17.5 inches from the wing root were computed. The computed results were compared with experimental results. The agreement was good, indicating that the difference equation approach holds promise as a means of determining the dynamic response of airplanes to shock loads.

The purpose of the present paper is to determine the effect of the use of finite time increments and of the replacement of the continuous structure by a discrete number of elastically connected point masses on the accuracy and convergence of numerical integration methods. The errors due to using too coarse a time increment to adequately describe the fine detail in the force-time history and due to approximating the initial conditions by various finite difference approximations are not considered in this

\footnotetext{
1 Figures in brackets indicate the literature references at the end of this paper.
}

report. A general study of errors in numerical integration procedures, using techniques similar to those in this report, is given in references [6] and [7].

\section{Error Due to Finite Time Increment}

In order to determine the errors introduced by finite time increments in numerical integration methods, a study is made of a single-degree-of-freedom system having simple loading conditions. The motion of more complicated systems can be considered as being made up of the motion in several normal modes, each mode acting as a single-degree-of-freedom system. The results of this study can be used, therefore, in judging the adequacy of the various numerical integration methods for complicated systems as well as for simple systems. The results are determined in analytical form, using the calculus of finite differences, for convenience in judging the accuracy of the methods and the peculiar nature of the errors introduced by using them.

\section{1. Basic Problem Considered}

The basic problem considered in this section is shown in figure 1. The mass, if initially disturbed, should vibrate without damping or amplitude buildup at a natural frequency $\omega=\sqrt{k / m}(\mathrm{rad} / \mathrm{sec})$. For such a system, the equation of motion is

$$
m \frac{d^{2} x}{d t^{2}}+k x=0
$$

In applying numerical integration methods to the solution of this equation, the following notation is used:

$\Delta t=$ time increment between successive steps in the numerical integration process.

$n=$ number of steps taken from $t=0$ to $t=n \Delta t$; as subscript, indicates value when $t=n \Delta t$.

$x_{n}=$ displacement when $t=n \Delta t$. 


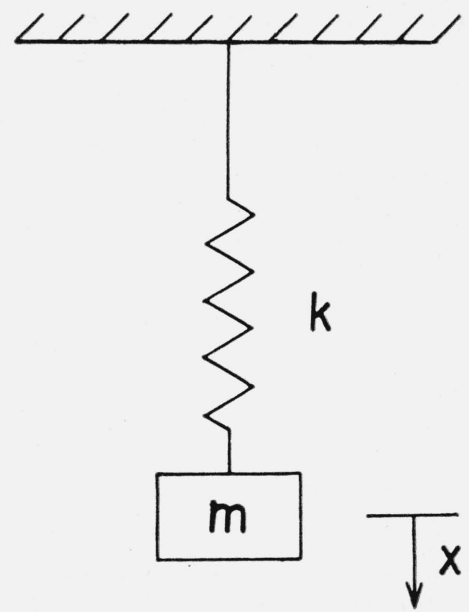

Figure 1. Basic problem considered in comparing numerical integration methods.

\subsection{Method Replacing Second Derivative by Equivalent Central Differences}

Substituting into eq (1)

$$
\left(\frac{d^{2} x}{d t^{2}}\right)_{n}=\frac{1}{(\Delta t)^{2}}\left(x_{n+1}-2 x_{n}+x_{n-1}\right)
$$

gives

$$
\frac{m}{(\Delta t)^{2}}\left(x_{n+1}-2 x_{n}+x_{n-1}\right)+k x_{n}=0,
$$

which reduces to

$$
x_{n+1}+\left[\frac{k(\Delta t)^{2}}{m}-2\right] x_{n}+x_{n-1}=0 .
$$

To solve eq (4) by the calculus of finite differences, we make the substitution,

$$
x_{n}=A \beta^{n},
$$

where $A$ is the arbitrary constant to be determined from initial conditions, and $\beta$ is the number to be so chosen that eq (4) is satisfied. Substituting from eq (5) into eq (4) gives

$$
A \beta^{n+1}+\left[\frac{k(\Delta t)^{2}}{m}-2\right] A \beta^{n}+A \beta^{n-1}=0 .
$$

Dividing through by $A \beta^{n-1}$, eq (6) reduces to

$$
\beta^{2}+\left[\frac{k(\Delta t)^{2}}{m}-2\right] \beta+1=0
$$

Several cases are of interest:

Case 2.2.1: $0<\Delta t<2 \sqrt{m / k}$; we will first consider $\Delta t=\sqrt{2} \sqrt{m / k}$; that is, $\Delta t=0.225(2 \pi) \sqrt{m / k}$, where
$(2 \pi) \sqrt{m / k}$ is the natural period of the system. In this case, eq (7) reduces to

from which,

$$
\beta^{2}+1=0,
$$

$$
\beta= \pm \sqrt{-1}= \pm i=e^{ \pm \frac{i_{\pi}}{2}} .
$$

Substituting this value of $\beta$ into eq (5),

$$
x_{n}=A e^{\frac{i \pi n}{2}}+B e^{-\frac{i \pi n}{2}}=A^{\prime} \sin ^{\frac{\pi n}{2}}+B^{\prime} \cos ^{\frac{\pi n}{2}},
$$

where $A, B, A^{\prime}, B^{\prime}$ are arbitrary constants determined from the initial conditions. Since

$$
n=t /(\Delta t)=t \sqrt{k / m} / \sqrt{2},
$$

eq (10) can be written

$$
x=A^{\prime} \sin 1.11 t \sqrt{k / m}+B^{\prime} \cos 1.11 t \sqrt{k / m} .
$$

In this case, the effect of the numerical integration method is to increase the effective natural frequency from $\sqrt{k / m}$ to $1.11 \sqrt{k / m}$ without introducing damping or buildup of the response.

As the value of the finite time increment $\Delta t$ is varied from 0 to $2 \sqrt{m / k}$, the factor 1.11 in eq (12) varies from 1 to $\pi / 2$, but otherwise the form of eq (12) does not change.

Case 2.2.2: $2 \sqrt{m / k}<\Delta t$; we will first consider $\Delta t=3 \sqrt{\mathrm{m} / \mathrm{k}}$. Although such a large value of $\Delta t$ would not ordinarily be used on a single-degree-offreedom system, it is usually impossible to avoid such a large value in a many-degree-of-freedom system having high frequency modes. With $\Delta t=3 \sqrt{\mathrm{m} / k}$, eq (7) becomes

$$
\beta^{2}+7 \beta+1=0
$$

giving

$$
\beta=-.1459=-e^{-1.925} ; \beta=-6.8541=-e^{1.925} .
$$

Substituting these values of $\beta$ into eq (5),

$x_{n}=A(-1)^{n} e^{-1.925 n}+B(-1)^{n} e^{1.925 n}$

$=A^{\prime} \cos n \pi \sinh 1.925 n+B^{\prime} \cos n \pi \cosh 1.925 n$.

Substituting $n=t / \Delta t=(t \sqrt{ } k / m) / 3$ into eq (15) gives

$$
\begin{aligned}
x= & {\left[\begin{array}{lll}
A^{\prime} & \sinh 0.642 t \sqrt{k / m}+B^{\prime} \cosh 0.642 t \sqrt{k / m}
\end{array}\right] } \\
& \cos 1.047 t \sqrt{k / m} .
\end{aligned}
$$

In this case, the primary effect of the numerical integration method is to introduce hyperbolic functions of time in the answer. Because these functions increase indefinitely with time, the solution is unstable. 
For all values of the time increment $\Delta t>2 \sqrt{m / k}$, unstable solutions of the type shown by eq (16) result. The larger the value of $\Delta t$, the greater will be the instability. It is this fact that makes the method considered in this section (2.2) inappropriate for use on many-degree-of-freedom systems where it is difficult to a void large values of $\Delta t$ relative to the higher frequency modes.

\subsection{Houbolt's Method}

If, in eq (1), Houbolt's substitution

$$
\left(\frac{d^{2} x}{d t^{2}}\right)_{n}=\frac{1}{(\Delta t)^{2}}\left(2 x_{n}-5 x_{n-1}+4 x_{n-2}-x_{n-3}\right)
$$

is made, we obtain

$$
\frac{m}{(\Delta t)^{2}}\left(2 x_{n}-5 x_{n-1}+4 x_{n-2}-x_{n-3}\right)+k x_{n}=0 .
$$

After transposing terms, eq (18) can be written as

$$
\left[2+\frac{k(\Delta t)^{2}}{m}\right] x_{n}-5 x_{n-1}+4 x_{n-2}-x_{n-3}=0 .
$$

Making use of the substitution given in eq (5), eq (19) becomes

$$
\left[2+\frac{k(\Delta t)^{2}}{m}\right] A \beta^{n}-5 A \beta^{n-1}+4 A \beta^{n-2}-A \beta^{n-3}=0 .
$$

Dividing through by $A \beta^{n-3}$ gives

$$
\left[2+\frac{k(\Delta t)^{2}}{m}\right] \beta^{3}-5 \beta^{2}+4 \beta-1=0 .
$$

Several cases are of interest.

Case 2.3.1: We will again consider $\Delta t=\sqrt{2} \sqrt{m / k}$, as was done in case 2.2.1. Equation (21) then reduces to

$$
4 \beta^{3}-5 \beta^{2}+4 \beta-1=0,
$$

giving the roots,

$$
\left.\begin{array}{l}
\beta=0.3710=e^{-0.991} \\
\beta=0.439+0.694 i=e^{(-0.197+1.007 i)} \\
\beta=0.439-0.694 i=e^{(-0.197-1.007 i)}
\end{array}\right\}
$$

Substitution of these values of $\beta$ into eq (5) gives

$$
\begin{aligned}
x_{n} & =A e^{-0.991 n}+B e^{(-0.197 n+1.007 i n)}+C e^{(-0.197 n-1.007 i n)} \\
& =A e^{-0.991 n}+e^{-0.197 n}\left(B^{\prime} \sin 1.007 n+C^{\prime} \cos 1.007 n\right)
\end{aligned}
$$

Using $n=t / \Delta t=(t \sqrt{k / m}) / \sqrt{2}$ gives

$$
\begin{aligned}
x= & A e^{-0.701 t \sqrt{k / m}}+e^{-0.139 t \sqrt{i / m}}\left(B^{\prime} \sin 0.712 t \sqrt{\frac{k}{m}}+\right. \\
& \left.C^{\prime} \cos 0.712 t \sqrt{\frac{k}{m}}\right)
\end{aligned}
$$

In this case, the effects of the numerical integration method used are (1) to introduce a decaying exponential, the first term; (2) to lower the natural frequency, 0.712 instead of 1 ; and (3) to introduce a damping term on the response, $e^{-0.139 t \sqrt{k / m}}$.

Case 2.3.2. The errors in the case where $\Delta$ is small can be judged best by a numerical example. With $\Delta t=\sqrt{0.1} \sqrt{m / k}$, a solution similar to that for case 2.3 .1 gives

$$
\begin{aligned}
x= & A e^{-2.32 t \sqrt{k / m}}+e^{-0.0095 t} \sqrt{k / m}\left(B^{\prime} \sin 0.961 t \sqrt{\frac{k}{m}}+\right. \\
& \left.C^{\prime} \cos 0.961 t \sqrt{\frac{k}{m}}\right) .
\end{aligned}
$$

In this case, the errors are a small drop in natural frequency, 0.961 in place of 1 ; and the presence of decaying exponentials. In the limit when $\Delta t \rightarrow 0$, the coefficient $2.32 \rightarrow \infty$; the coefficient $0.0095 \rightarrow 0$; and the coefficient $0.961 \rightarrow 1$. The solution approaches the exact solution as $\Delta t$ approaches zero.

Case 2.3.3. To judge the errors for large values of $\Delta t$, we will consider the numerical case where $\Delta t=$ $\sqrt{98} \sqrt{m / k}$. In this case, the solution is given by

$$
\begin{aligned}
x= & A e^{-0.1805 t \sqrt{k / m}}+e^{-0.1424 t} \sqrt{k / m}\left(B^{\prime} \sin 0.183 t \sqrt{\frac{k}{m}}+\right. \\
& \left.C^{\prime} \cos 0.183 t \sqrt{\frac{k}{m}}\right) .
\end{aligned}
$$

The errors are a large drop in natural frequency, 0.183 in place of 1 , and the presence of decaying exponentials. In the limit when $\Delta t \rightarrow \infty$, the coefficients $0.1805,0.1424$, and 0.183 all approach zero. It is thus seen that large errors may result for large values of $\Delta t$, but that they will always be of a stable type; rounding-off errors will not build up.

\section{Error Due to Replacing Continuous Struc- ture by a Discrete Number of Elastically Connected Masses}

An airplane wing is, essentially, a beam and, like a beam, has an infinite number of degrees of freedom. Since an analysis of such a structure without simplification be difficult, if not impossible, it is customary to replace the distributed mass of the airplane by lumped masses connected by massless springs chosen, appendix II of reference [8], to approximately represent the large-scale motions of the airplane. It is also customary to approximate the distributed mass by generalized masses [1], corresponding to the lower modes of vibration. Such lumped or generalized masses are never equivalent in their behavior to the distributed mass of the airplane, since they can only represent a finite number of degrees of freedom.

A general investigation of errors resulting from the use of a finite number of masses is beyond the scope of this report; however, since the Houbolt method has advantages insofar as numerical integration is 


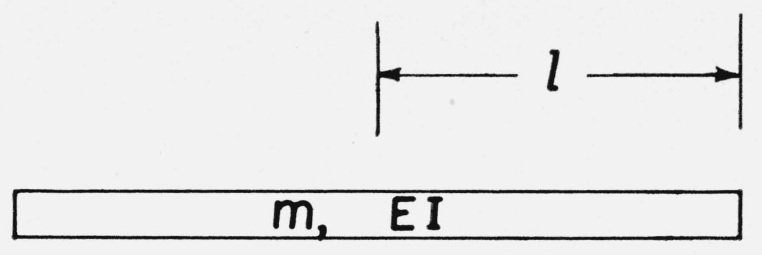

Figure 2. Beam used in computations.

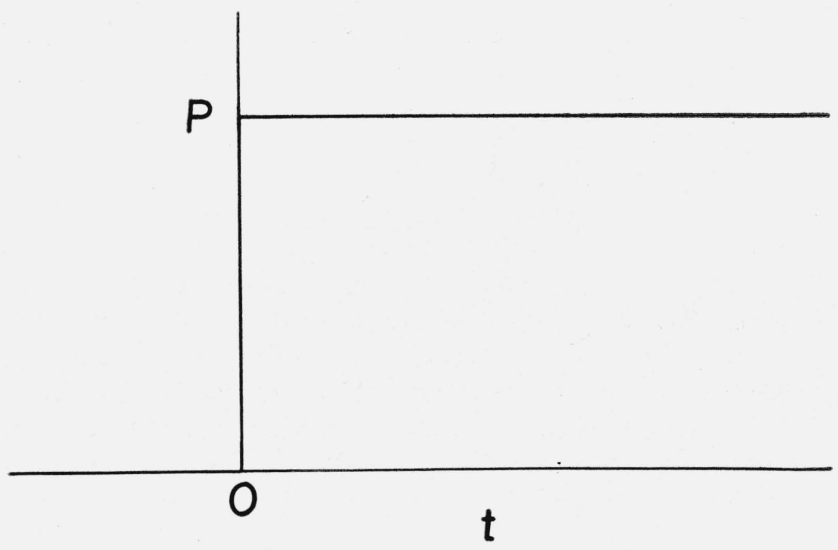

Figure 3. Time history of force applied to beam.

concerned, a study is made of the error resulting when this method of numerical integration is used and a finite number of lumped masses replace the distributed mass. This is done by comparing the computed responses with 3,5 , and 7 lumped masses for an undamped, uniform, free-free beam of mass $m$, length $2 l$ and bending stiffness, $E I$, figure 2 , using Houbolt's method as modified in reference [5]. It is assumed that the beam is subjected to suddenly applied transverse loads of the type shown in figure 3. As more complicated time histories of loading can be considered to be made up of stepwise changes of the type shown in figure 3, the results will be significant for any type of transverse loading. All displacements are taken in a vertical direction. Translation and rotation of the beam are considered in addition to bending.

\subsection{Uniform Beam With Load Applied at Center}

A uniform beam is considered to be suddenly subjected to a constant normal force $P$ at its center. The beam is idealized by considering the mass of the actual beam, figure 4 (a), to be concentrated at 3-, 5-, and 7-mass points, figures 4 (b), 4 (c), and 4 (d), respectively.

To evaluate the combined errors due to the use of finite numbers of mass points and finite time increments, three time increments are used in the numerical analysis for each mass distribution. These time increments are

$\Delta t=\sqrt{m l^{3} / 12 E I}, \quad \frac{1}{3} \sqrt{m l^{3} / 12 E I}, \quad$ and $\frac{1}{5} \sqrt{m l^{3} / 12 E I}$.

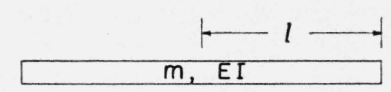

(a) Actual beam

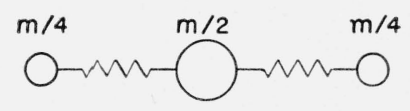

(b) Three mass system

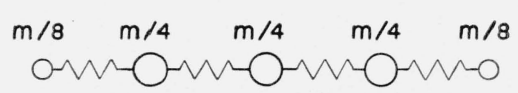

(c) Five mass system

$\begin{array}{llllllll}m / 12 & m / 6 & m / 6 & m / 6 & m\end{array} / 6 \quad m / 6 \quad m / 12$

On-Om-Om-Om-Om-Omo

(d) Seven mass system

Figure 4. Distributions of mass considered for beam subjected to impact at center.

The computations are carried out as described in reference [5] and as shown for a typical case in the appendix. In this procedure, the initial conditions are taken into account by considering the displacements zero for three time increments prior to the initial instant of time. The flexibility is taken into account by the use of influence coefficients. Matrix algebra is used to simultaneously compute the displacements at all the mass points at a particular increment of time from the given forces acting at that time and from the known displacements at the preceding three increments in time. The bending moment at the center is then computed from the inverse of the influence coefficient matrix, the displacements at the various mass points, and the lever arms from these mass points to the center of the beam. The bending moment, $M$, at the center of the beam, positive when concave away from the side of the beam being struck, is plotted on a dimensionless basis in figures 5, 6, and 7 for the 3-, 5-, and 7mass approximations, respectively. It is noted that, as the time interval used in the step-by-step numerical integration is shortened, the maximum absolute value of the bending moment is increased. As is to be expected from the analysis of a single degree-of-freedom system, the errors of the numerical method cause a damping out of the oscillatory response and, as the time increment is decreased, a decrease in the period of the oscillatory response.

For each of figures 5 to 7 , the mass distribution is kept constant, and the increment of time used in the numerical integration is varied to show the effect of time increment. In each of figures 8 to 10 , the time increment is kept constant, and the responses for the different mass distributions are plotted to bring out more clearly the effect of increasing the 


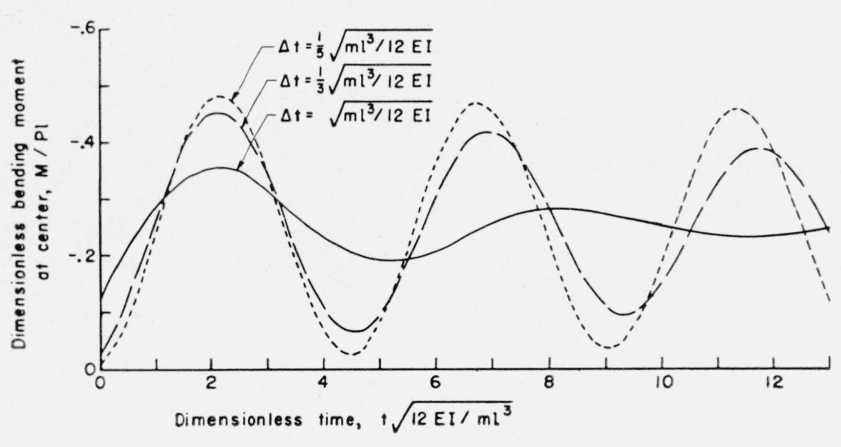

Figure 5. Bending moment ratio at the center of the beam caused by impact at center, three-mass idealization.

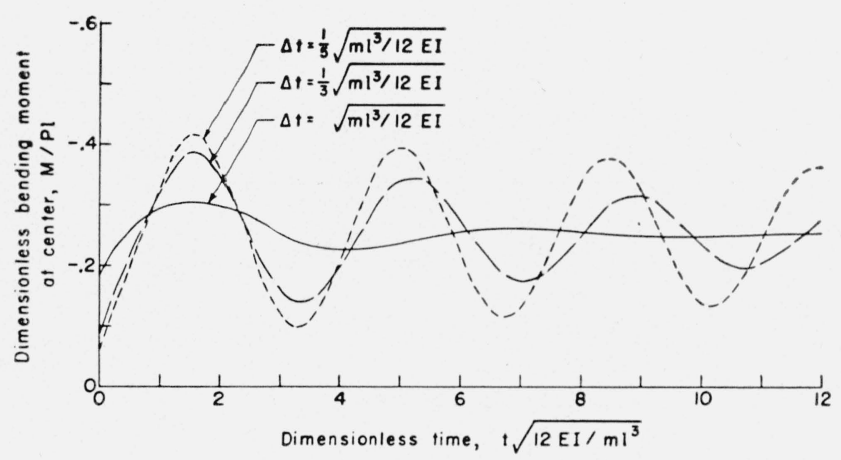

Figure 6. Bending moment ratio at the center of the beam caused by impact at center, five-mass idealization.

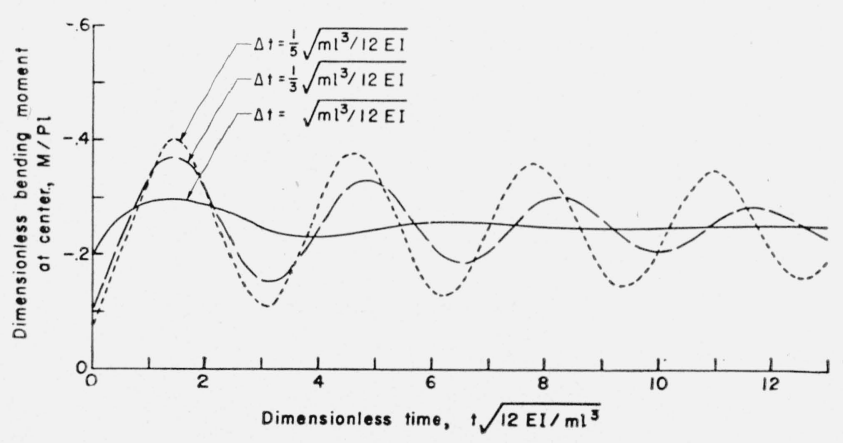

Figure 7. Berding moment ratio at the center of the beam caused by impact at center. seven-mass idealization.

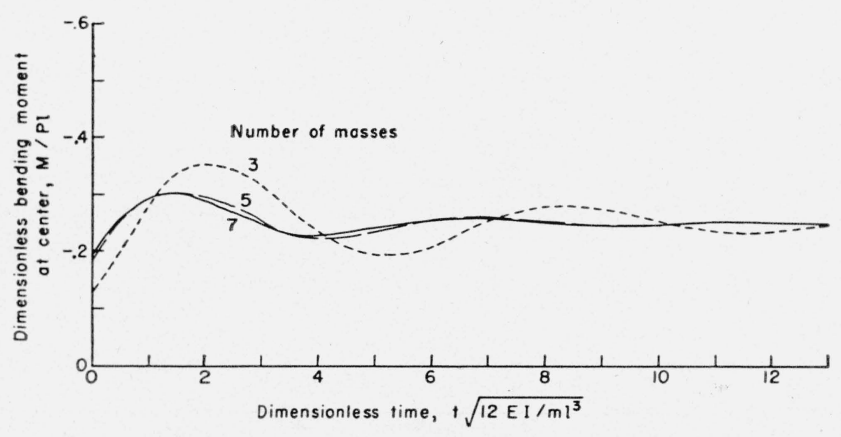

Figure 8. Bending moment ratios for different mass idealizations with impact at center mass, time increment $\Delta t=\sqrt{m l^{3} / 12 E I}$.

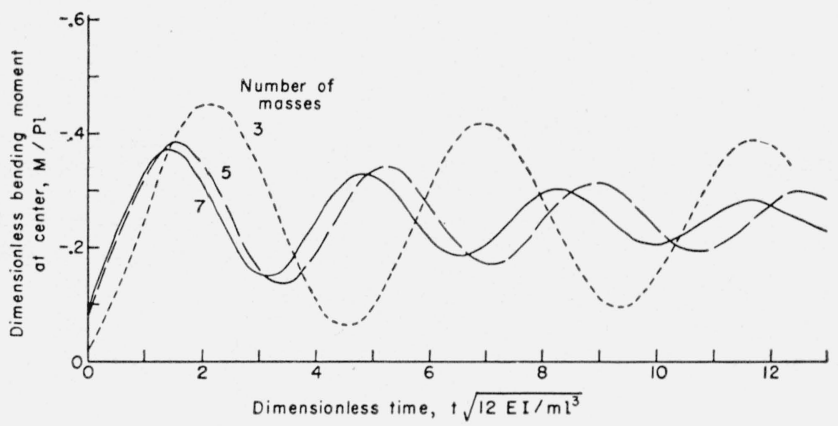

Figure 9. Bending moment ratios for different mass idealizations with impact at center mass, time increment $\Delta t=1 / 3 \sqrt{m l^{3} / 12 E I}$.

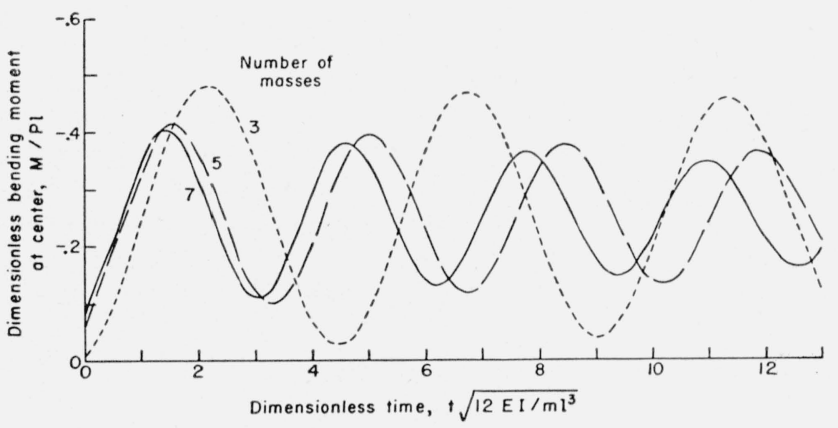

Figure 10. Bending moment ratios for different mass idealizations with impact at center mass, time increment $\Delta t=1 / 5 \sqrt{m l^{3} / 12 E I}$.

number of masses. The maximum absolute value of the bending moment at the center differs by not more than 4 percent for the 5 - and 7 -mass systems, regardless of time increment. There is some difference in the period of the response for the shorter time increments for the 5- and 7-mass systems.

\subsection{Beam With Impact at One Tip}

Because it was believed that an impact at a tip mass might result in a more severe loading condition, an investigation is made for the case of a load $P$ applied at the tip of the beam shown in figure 4 (a), using the idealizations shown in figures 4 (b) and 4 (c). The bending moments at the center for the 3 - and 5-mass idealizations are shown in figures 11 and 12 , respectively. It is noted again, as in case 3.1 , that as the time interval used in the step-bystep numerical integration is shortened, the maximum absolute value of the bending moment at the center is increased. As is to be expected from the analysis of a single-degree-of-freedom system, the errors of the numerical method cause a damping out of the oscillations, which is particularly evident for the longest time increment, and a decrease in the period of the oscillatory response as the time increment is decreased.

In figures 13 to 15 , the time increment is kept constant, and the responses for idealization of the beam by 3 and 5 masses are plotted to bring out 


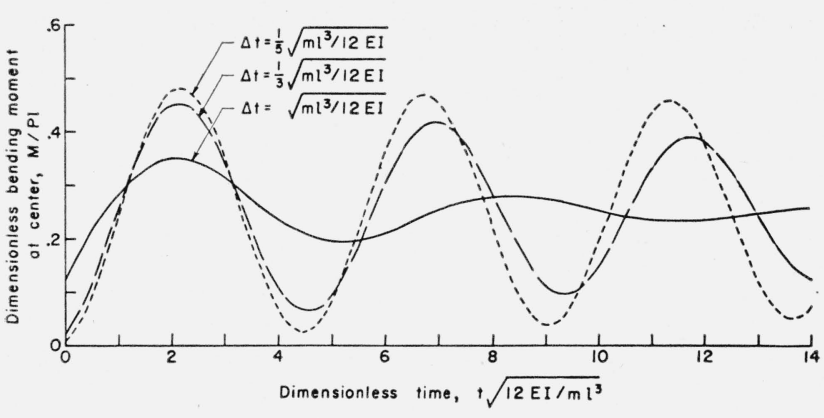

PIgUkE 11. Bending moment ratio at the center of the beam caused by impact at a tip, three-mass idealization.

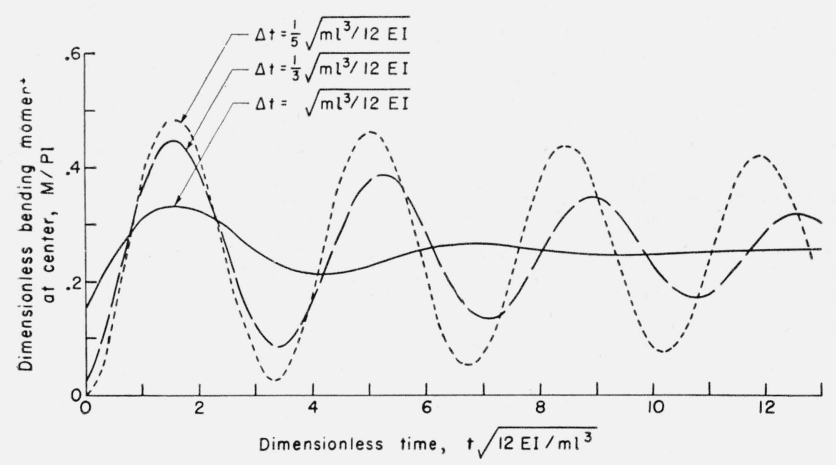

Figure 12. Bending moment ratio at the center of the beam caused by impact at a tip, five-mass idealization.

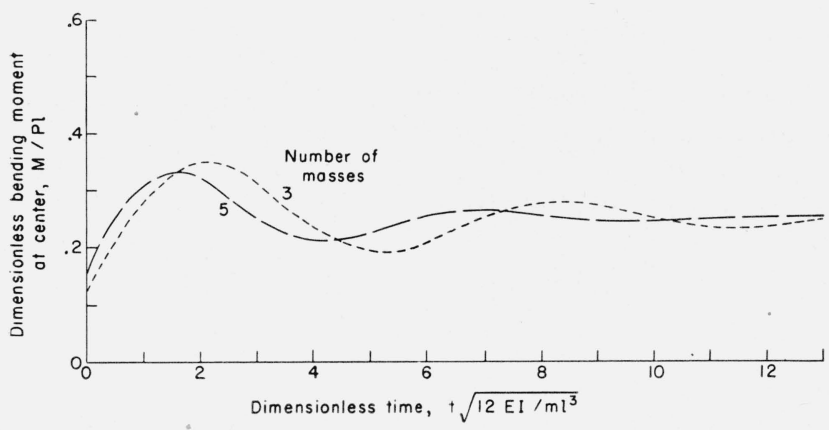

FIGURE 13. Bending moment ratios for different mass idealizations with impact at tip, time increment $\Delta t=\sqrt{m l^{3} / 12 E I}$.

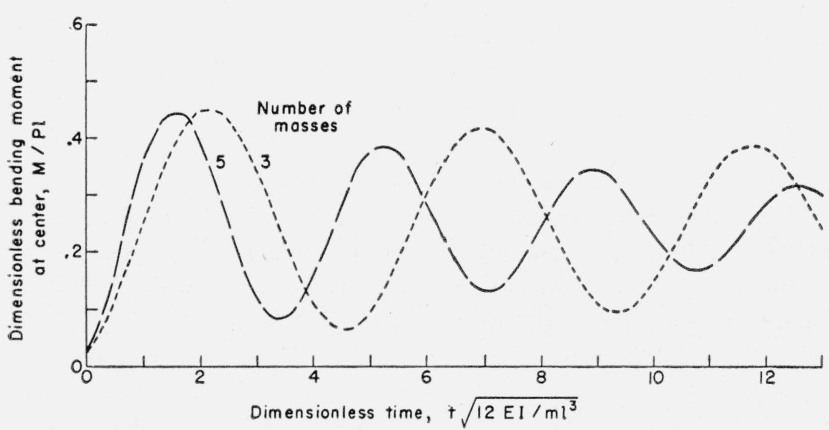

Figure 14. Bending moment ratios for different mass idealizations with impact at tip, time increment $\Delta t=1 / 3 \sqrt{m l^{3} / 12 E I}$.

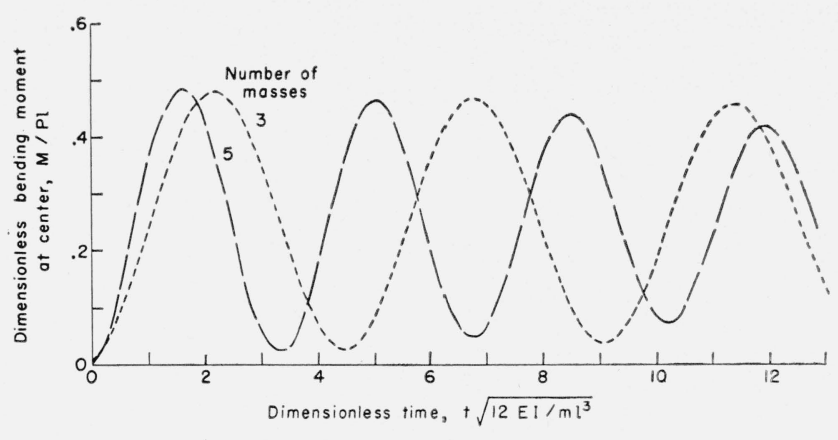

FIGURE 15. Bending moment ratios for different mass idealizations with impact at tip, time increment $\Delta t=1 / 5 \sqrt{m l^{3} / 12 E I}$.

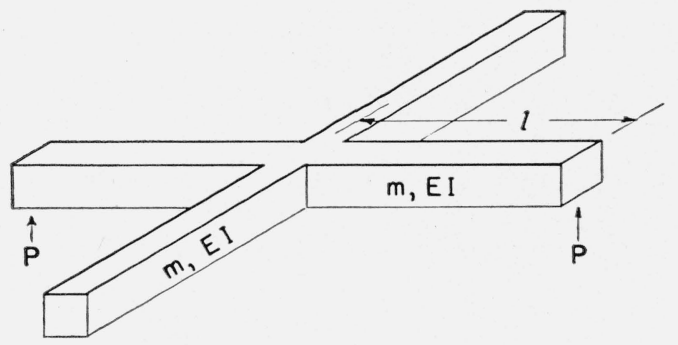

(a) Actual beams

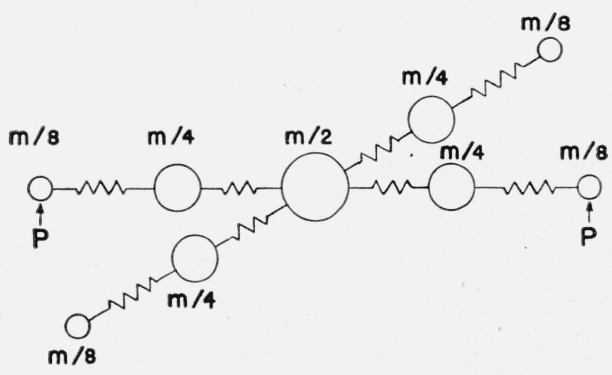

(b) Idealized beams

Figure 16. Mass distribution considered for two beams normal to each other subjected to tip impact.

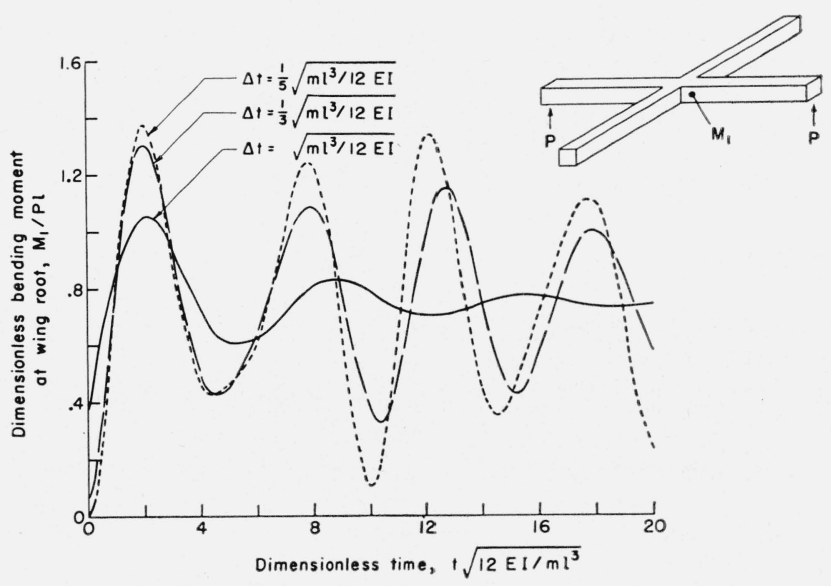

Figure 17. Bending moment at wing root for pair of beams representing airplane, each beam idealized by five masses. 


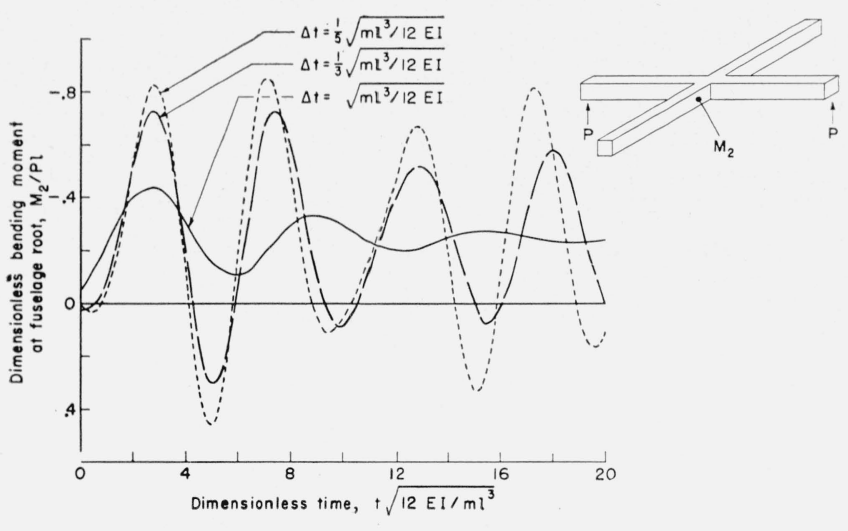

Figure 18. Bending moment at fuselage root for pair of beams representing airplane, each beam idealized by five masses.

the effect of increasing the number of masses. It can be seen that the period of the initial vibratory response decreases with an increase in the number of masses and that the damping increases. The maximum bending moment at the center is relatively unaffected.

\subsection{Pair of Beams Representing a Fuselage and Wing With Impact at Both Wing Tips}

An airplane can be approximated by two uniform beams at right angles to each other, one beam representing the wing, the other the fuselage. Such a system, figure 16 (a), is investigated. Each beam is idealized as 5 masses, as shown in figure 16 (b). The suddenly applied constant loads, $P$, are considered to act at each wing tip. The bending moments at the center of both the "fuselage" and "wing" beams are computed by using the same three time increments in the numerical integration as were previously used. The bending moment at the center of the struck beam (wing) is shown in figure 17 and that for the other beam (fuselage) is shown in figure 18. The agreement between the bending moment ratios for the three values of time increment is only fair even for the two shortest time increments. As might have been expected from the analysis given of the single-degree-of-freedom system, the error due to using the longest time increment is evident in the marked damping of the oscillatory response.

\subsection{Discussion}

It is estimated that a time increment of about $1 / 30$ of the period in the fundamental mode is necessary for good accuracy in numerical-integration methods. This value is based on several factors.

(1) The solution given in eq (26) corresponds to about 20 time increments per fundamental period. In this case the frequency coefficient, 0.961, is in error by about 4 percent.

(2) The convergence indicated in the figures is not quite complete for the shortest time increment, which corresponds to $1 / 13.74$ of the fundamental symmetrical mode period and only $1 / 5.00$ of the lowest antisymmetrical mode period. A shorter time increment than this is certainly needed to give the response of the higher modes.

(3) The recommended time increment is likely to be used for computing responses with slower convergence than the bending moment at the center of the beam and for structures with slower convergence than that of a uniform beam.

(4) It is likely that computations by numerical methods will be carried out by automatic computing machines where the advantage of additional accuracy from a smaller time increment can be had with less penalty than when hand computing is used.

It is noted from figures 8,9 , and 10 that, for each time increment considered, the response to an impact at the center is essentially the same for the 5- and 7 -mass idealizations of the beam but considerably higher for the 3-mass system. For impacts at one end of the beam, figures 13,14 , and 15 , the response shows less change in going from the 3 - to the 5-mass idealizations than is the case for impact at the center. It would appear that a uniform beam should be approximated by a 5-mass idealization if errors in the bending moment at the center are to be small.

In table 1, the maximum absolute values of bending moment ratios are given. For the beam with the impact at the center, the absolute value of the bending moment increased as the time interval for integration was decreased, but decreased as the number of masses used for the idealization of the distributed mass of the beam was increased. From this, it may well be that the errors caused by using a reasonable value of time interval for the numerical integration will be offset to some extent by the errors caused by using a finite number of masses. For the impact at a tip mass, the increase in bending moment with decrease in time interval is appreciable, but the decrease in bending moment with increase in number of masses is much less than for the case of impact at the center.

TABLE 1. Maximum absolute value of bending moment at center of uniform beam struck with force $P$

\begin{tabular}{|c|c|c|c|}
\hline \multirow{2}{*}{ Time interval } & \multicolumn{3}{|c|}{$|\boldsymbol{M} / P l|$} \\
\hline & 3 -mass & 5 -mass & 7 -mass \\
\hline \multicolumn{4}{|c|}{ Impact at center } \\
\hline$\sqrt{m l^{3 / 12 E I}}$ & 0.355 & 0.305 & 0.295 \\
\hline $1 / 3 \sqrt{m l^{3} / 12 E I}$ & .450 & .383 & .368 \\
\hline $1 / 5 \sqrt{m l^{3} / 12 E I}$ & .480 & .416 & .400 \\
\hline \multicolumn{4}{|c|}{ Impact at tip } \\
\hline$\sqrt{m l^{3 / 12 E I}}$. & 0.355 & 0.333 & \\
\hline $1 / 3 \sqrt{m l^{3} / 12 E I}$ & .450 & .448 & -... \\
\hline $1 / 5 \sqrt{m l^{3} / 12 E I_{-}}$ & .480 & .485 & \\
\hline
\end{tabular}


It is evident from figure 5 that the period of the response approaches the value for the exact solution for a beam idealized by 3 masses as the time interval in the integration is shortened. This same decrease in period with time increment is noted in figures 6 and 7 for the 5- and 7 -mass idealization of the beam. The period of the response also decreases as the number of masses is increased, figures 7,8 , and 9 . This latter result, at least qualitatively, verifies that obtained by Duncan [9]. He has obtained an exact solution showing that the periods of the computed modes of vibration of a uniform beam decrease as the number of masses chosen to represent the beam is increased, and that the magnitudes of the errors in the computed periods vary inversely as the square of the number of masses.

\section{Conclusions}

From the analyses of the responses of single-degreeof-freedom and many-degree-of-freedom systems to a suddenly applied force, it may be concluded that:

a) Numerical integration methods give results that closely approximate the exact solution only if the increment of time between successive steps in the integration is small compared to all the natural periods of vibration of the system. In cases similar to those investigated where bending moment near the center is desired, a time increment of about $1 / 30$ of the fundamental period of the system will give good results.

b) Of the methods investigated, the Houbolt method is the only one that gives convergent results for large time increments. In this method, errors introduced by large time increments result in the damping out of oscillatory response.

c) The distributed mass of the beam can be considered to be concentrated at relatively few mass points for computational purposes. In cases similar to those investigated, approximating a uniform beam by a 5-mass idealization gives good results.

\section{Appendix}

The detailed method of determining the response of a uniform beam to an impact load by numerical integration is given in this appendix for the case of a beam subjected to an impact at its tip. The case considered is that where the time increment is $\Delta t=\sqrt{m l^{3} / 12 E I}$. The responses for all the other cases presented in this report are obtained in a similar manner.

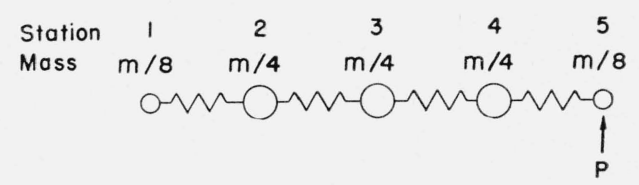

Figure 19. Mass distribution for example considered in appendix.
The mass of the uniform beam is considered to be distributed at five stations along its length, figure 19 . The force, $P$, is applied at the tip, station 5. As used in this report, an influence coefficient, $\delta_{r, s}$ is the displacement at station $r$ due to a unit load at station $s$ with the center of the beam, station 3 , fixed. The influence coefficients are given in table 2. Denoting the displacement positive upward at station $r$ by $d_{\tau}$, the force at station $r$ by $F_{r}$, and the rotation at station 3 by $\alpha$, the displacements of the beam at stations 1 , 2,4 , and 5 are

$$
\left.\begin{array}{l}
d_{1}=d_{3}+\delta_{11} F_{1}+\delta_{12} F_{2}-\alpha l, \\
d_{2}=d_{3}+\delta_{12} F_{1}+\delta_{22} F_{2}-\alpha \frac{l}{2}, \\
d_{4}=d_{3}+\delta_{44} F_{4}+\delta_{45} F_{5}+\alpha \frac{l}{2}, \\
d_{5}=d_{3}+\delta_{45} F_{4}+\delta_{55} F_{5}+\alpha l
\end{array}\right\}
$$

Substituting the values of the influence coefficients from table 2 into eq (A1), and solving for the forces

\begin{tabular}{|c|c|c|c|c|c|}
\hline & 1 & 2 & 3 & 4 & 5 \\
\hline $\begin{array}{l}1 \\
2 \\
3 \\
4 \\
5\end{array}$ & $\begin{array}{c}l^{3 / 3} / 3 E I \\
5 l^{3} / 48 E I \\
0 \\
0 \\
0\end{array}$ & $\begin{array}{c}5 l^{3} / 48 E I \\
l 3 / 24 E I \\
0 \\
0 \\
0\end{array}$ & $\begin{array}{l}0 \\
0 \\
0 \\
0 \\
0\end{array}$ & $\begin{array}{c}0 \\
0 \\
0 \\
l^{3} / 24 E I \\
5 l^{3} / 48 E I\end{array}$ & $\begin{array}{c}0 \\
0 \\
0 \\
5 l^{3 / 48 E I} \\
l^{3} / 3 E 1\end{array}$ \\
\hline
\end{tabular}

TABLE 2. Influence coefficients $\delta_{r, s}$ for 5 -mass idealized beam shown in figure 19

in terms of the displacements at each station and of the rotation at station 3 .

$$
\left.\begin{array}{l}
F_{1}=\frac{48 E I}{7 l^{3}}\left(2 d_{1}-5 d_{2}+3 d_{3}-\frac{\alpha l}{2}\right), \\
F_{2}=\frac{48 E I}{7 l^{3}}\left(-5 d_{1}+16 d_{2}-11 d_{3}+3 \alpha l\right), \\
F_{4}=\frac{48 E I}{7 l^{3}}\left(-11 d_{3}+16 d_{4}-5 d_{5}-3 \alpha l\right), \\
F_{5}=\frac{48 E I}{7 l^{3}}\left(3 d_{3}-5 d_{4}+2 d_{5}+\frac{\alpha l}{2}\right)
\end{array}\right\} .
$$

From the condition that the sum of the forces equal zero,

$$
F_{1}+F_{2}+F_{3}+F_{4}+F_{5}=0,
$$

and substituting from eq (A2), the value of $F_{3}$ is

$$
F_{3}=\frac{48 E I}{7 l^{3}}\left[3 d_{1}-11 d_{2}+16 d_{3}-11 d+3 d_{3}\right]
$$


From the requirement that the net moment equal zero,

$$
F_{1}(0)+F_{2}\left(\frac{l}{2}\right)+F_{3}(l)+F_{4}\left(\frac{3 l}{2}\right)+F_{5}(2 l)=0,
$$

and using eq (A2) and (A4),

$$
\alpha=0.25 \frac{d_{1}}{l}-1.5 \frac{d_{2}}{l}+1.5 \frac{d_{4}}{l}-0.25 \frac{d_{5}}{l} .
$$

Substituting the value of $\alpha$ from eq (A6) into eq (A2), and rewriting eq (A4):

$$
\left.\begin{array}{l}
F_{1}=\frac{48 E I}{7 l^{3}}\left(1.875 d_{1}-4.25 d_{2}+3.00 d_{3}-0.75 d_{4}+0.125 d_{5}\right), \\
F_{2}=\frac{48 E I}{7 l^{3}}\left(-4.25 d_{1}+11.50 d_{2}-11.00 d_{3}+4.50 d_{4}-0.75 d_{5}\right), \\
F_{3}=\frac{48 E I}{7 l^{3}}\left(3.00 d_{1}-11.00 d_{2}+16.00 d_{3}-11.00 d_{4}+3.00 d_{5}\right), \\
F_{4}=\frac{48 E I}{7 l^{3}}\left(-0.75 d_{1}+4.50 d_{2}-11.00 d_{3}+11.50 d_{4}-4.25 d_{5}\right), \\
F_{5}=\frac{48 E I}{7 l^{3}}\left(0.125 d_{1}-0.75 d_{2}+3.00 d_{3}-4.25 d_{4}+1.875 d_{5}\right)
\end{array}\right\}(\mathrm{A} 7)
$$

The forces are the sum of the inertia loads due to the acceleration of the mass of the beam and the applied forces,

$$
\left.\begin{array}{l}
F_{1}=-\frac{m}{8} \ddot{d}_{1}, \\
F_{2}=-\frac{m}{4} \ddot{d}_{2}, \\
F_{3}=-\frac{m}{4} \ddot{d}_{3}, \\
F_{4}=-\frac{m}{4} \ddot{d}_{4}, \\
F_{5}=-\frac{m}{8} \ddot{d}_{5}+P
\end{array}\right\},
$$

where $m$ is mass of uniform beam; $\ddot{d}_{\tau}$, acceleration of beam at station $r(r=1,2, \ldots 5)$; and $P$, applied constant force at station 5 .

Using the difference eq (17), acceleration at station $r$ can be expressed as

$\left(\ddot{d}_{r}\right)_{t}=\frac{2}{\Delta t^{2}}\left(d_{r}\right)_{t}-\frac{5}{\Delta t^{2}}\left(d_{r}\right)_{t-\Delta t}+\frac{4}{\Delta t^{2}}\left(d_{r}\right)_{t-2 \Delta t}+\frac{1}{\Delta t^{2}}\left(d_{r}\right)_{t-3 \Delta t}$.

Equating the first of eq $(\mathrm{A} 7)$ and $(\mathrm{A} 8)$ and substituting for $\left(\ddot{d}_{r}\right)_{t}$ from eq (A9), we obtain

$$
\begin{aligned}
& -\frac{m}{8 \Delta t^{2}}\left[2\left(d_{1}\right)_{t}-5\left(d_{1}\right)_{t-\Delta t}+4\left(d_{1}\right)_{t-2 \Delta t}-\left(d_{1}\right)_{t-3 \Delta t}\right]= \\
& \quad \frac{48 E I}{7 l^{3}}\left[1.875 d_{1}-4.25 d_{2}+3.00 d_{3}-0.75 d_{4}+0.125 d_{5}\right]_{t} .
\end{aligned}
$$

Similar relations are obtained by using the remaining eq (A7) and (A8).

Multiplying eq (A10) and the corresponding equations for the other stations through by $3 E I / \mathrm{Pl}^{3}$ to express the deflections as a dimensionless ratio, and transposing terms, the equations can be written in a form which, for station 1, reads

$$
\begin{gathered}
\left(1.875+\frac{7 m l^{3}}{192 \Delta t^{2}}\right)\left(\frac{3 d_{1} E I}{P l^{3}}\right)_{t}-4.25\left(\frac{3 d_{2} E I}{P l^{3}}\right)_{t}+ \\
3.00\left(\frac{3 d_{3} E I}{P l^{3}}\right)_{t}-0.75\left(\frac{3 d_{4} E I}{P l^{3}}\right)_{t}+ \\
0.125\left(\frac{3 d_{5} E I}{P l^{3}}\right)_{t}=\left(J_{1}\right)_{t},
\end{gathered}
$$

where

$$
\begin{aligned}
\left(J_{1}\right)_{t}= & \frac{7 m l^{3}}{384 E I \Delta t^{2}}\left[5\left(\frac{3 d_{1} E I}{P l^{3}}\right)_{t-\Delta t}-\right. \\
& \left.4\left(\frac{3 d_{1} E I}{P l^{3}}\right)_{t-2 \Delta t}+\left(\frac{3 d_{1} E I}{P l^{3}}\right)_{t-3 \Delta t}\right] .
\end{aligned}
$$

Equation (A11) and the equations similar to it can be expressed in matrix form as

$$
[A]\left\{\frac{3 d_{r} E I}{P l^{3}}\right\}_{t}=\left\{J_{r}\right\},
$$

where the matrix $[A]$ is given in table 3 . Solving eq (A13) for the deflections gives,

$$
\left\{\frac{3 d_{r} E I}{P l^{3}}\right\}_{t}=[A]^{-1}\left\{J_{\tau}\right\}_{t}
$$


Taвle 3. Matrix $[A]$ of deflection coefficients for use in equation $\left(A 13^{\prime}\right)$

\begin{tabular}{|c|c|c|c|c|}
\hline \hline $1.875+\frac{7 m l^{3}}{192 E I \Delta t^{2}}$ & -4.25 & 3.00 & -0.75 & 0.125 \\
-4.25 & $11.50+\frac{7 m l^{3}}{96 E I \Delta t^{2}}$ & -11.00 & 4.50 & -.75 \\
3.00 & -11.00 & $16+\frac{7 m l^{3}}{96 E I \Delta t^{2}}$ & -11.00 & 3.00 \\
-0.75 & 4.50 & -11.00 & $11.5+\frac{7 m l^{3}}{96 E I \Delta t^{2}}$ & -4.25 \\
.125 & -0.75 & 3.00 & -4.25 & $1.875+\frac{7 m l^{3}}{192 E I \Delta t^{2}}$ \\
\hline
\end{tabular}

where $[A]^{-1}$ is obtained as the inverse of $[A]$ with $\Delta t=\sqrt{m l^{3} / 12 E I}$. The matrix $[A]^{-1}$ is given in table 4 . The first of this set of equations in conventional form is

$$
\begin{aligned}
& \left(\frac{3 d_{1} E I}{P l^{3}}\right)_{t}=1.344937\left(J_{1}\right)_{t}+0.589567\left(J_{2}\right)_{t}+ \\
& 0.113294\left(J_{3}\right)_{t}-0.113732\left(J_{4}\right)_{t}-0.237485\left(J_{5}\right)_{t},
\end{aligned}
$$

where, from eq (A12),

$$
\begin{array}{r}
\left(J_{1}\right)_{t}=1.09375\left(\frac{3 d_{1} E I}{P l^{3}}\right)_{t-\Delta t}-0.87500\left(\frac{3 d_{1} E I}{P l^{3}}\right)_{t-2 \Delta t} \\
+0.21875\left(\frac{3 d_{1} E I}{P l^{3}}\right)_{t-3 \Delta t} \cdot \quad(\mathrm{A} 16)
\end{array}
$$

Similar equations can be obtained for $J$ at the other stations.

TABLE 4. Inverted matrix $[A]^{-1}$ of table 3 with $\Delta t=\sqrt{m l^{3} / 12 E I}$

\begin{tabular}{|r|r|r|r|r|}
\hline & & & \\
1.344937 & 0.589567 & 0.113294 & -0.113732 & -0.237485 \\
0.589567 & .519302 & .305894 & .079741 & -.113732 \\
.113294 & .305894 & .417773 & .305894 & .113294 \\
-.113732 & .079741 & .305894 & .519302 & .589567 \\
-.237485 & -.113732 & .113294 & .589567 & 1.344937 \\
\hline
\end{tabular}

It is assumed that, prior to the application of the force, $P$, the displacements at all stations are zero. The computations are tabulated as shown in tables 5 and 6 . The $J$ 's are computed for time $t$, table 6 , using eq (A16) for $\left(J_{1}\right)_{t}$ and similar equations for the other $J$ 's. The $d$ 's are computed for time $t$, table 5, using eq $(\mathrm{A} 15)$ for $\left(3 d_{1} E I / P l^{3}\right)_{t}$ and similar equations for the other $d$ 's. From the $d$ 's at time $t$, the J's at time $t+\Delta t$ are computed and, from these, the $d$ 's at time $t+\Delta t$. Thus a time history of the displacement ratios of the uniform beam due to the suddenly applied constant force is obtained.

Since the step-by-step numerical procedure is dependent on previously computed values, it is desirable to have a check column which will indicate errors made in the computation. The deflections at each station were checked by computing the displacement, $D$, of the center of gravity of the beam from two different formulas. If these two values of $D$ agreed, the computation of the deflections was assumed correct. The first equation for $D_{t}$ is:

$$
\frac{3 E I}{P l^{3}} D_{t}=\frac{1}{m} \sum_{r=1}^{5}\left(m_{r} \frac{3 d_{r} E I}{P l^{3}}\right)_{t}
$$

where

$$
\begin{aligned}
& m=\sum_{r=1}^{5} m_{r} \\
& \frac{3 E I}{P l^{3}} D_{t}=0.125\left(\frac{3 d_{1} E I}{P l^{3}}\right)_{t}+0.250\left(\frac{3 d_{2} E I}{P l^{3}}\right)_{t}+ \\
& 0.250\left(\frac{3 d_{3} E I}{P l^{3}}\right)_{t}+0.250\left(\frac{3 d_{4} E I}{P l^{3}}\right)_{t}+ \\
& 0.125\left(\frac{3 d_{5} E I}{P l^{3}}\right)_{t} \text {. }
\end{aligned}
$$

The other equation is obtained by substituting for $\left[\left(3 d_{1} E I\right) / P l^{3}\right]_{t}, \quad\left[\left(3 d_{2} E I\right) / P l^{3}\right]_{t}, \quad$. . . . $\left[\left(3 d_{5} E I\right) / P l^{3}\right]_{t}$ in eq (A19), their equivalents in terms of the $J$ 's eq (A14). This gave

$$
\begin{aligned}
\frac{3 E I}{P l^{3}} D_{t}= & 0.28571375\left(J_{1}\right)_{t}+0.28571362\left(J_{2}\right)_{t}+ \\
& 0.28571375\left(J_{3}\right)_{t}+0.28571362\left(J_{4}\right)_{t}+ \\
& 0.28571375\left(J_{5}\right)_{t} .
\end{aligned}
$$

The small differences in the coefficients in eq (A20) result from rounding-off error. For a good error indication, the coefficients, as given, are needed. The values of $D$ from eq (A19) and (A20) are given in columns (7) and (8) of table 5.

The $J$ 's are checked by comparing their sums as determined from two different formulas. The first formula is

$$
\left(\sum_{r=1}^{5} J_{r}\right)_{t}=\left(J_{1}\right)_{t}+\left(J_{2}\right)_{t}+\left(J_{3}\right)_{t}+\left(J_{4}\right)_{t}+\left(J_{5}\right)_{t}
$$

The second formula is obtained by substituting in eq (A21) the value of $\left(J_{1}\right)_{t}$ in eq (A16) and corresponding values for the other $J$ 's. Then, making use of eq (A19),

$$
\begin{gathered}
\left(\sum_{r=1}^{5} J_{r}\right)_{t}=8.75(D)_{t-\Delta t}-7.00(D)_{t-2 \Delta t}+ \\
1.75(D)_{t-3 \Delta t}+0.4375
\end{gathered}
$$

The values computed from eq (A21) and (A22) are given in columns (7) and (8), respectively, of table 6 . The good agreement between the check columns in tables 5 and 6 indicate that the computation is free of numerical error. 
TABLE 5. Displacement ratios at stations along a uniform beam subjected to an impact at its tip

\begin{tabular}{|c|c|c|c|c|c|c|c|c|}
\hline 1 & 2 & 3 & 4 & 5 & 6 & 7 & 8 & 9 \\
\hline \multirow{2}{*}{$\begin{array}{l}\text { Time } \\
\text { ratio } \\
t \sqrt{\frac{12 E I}{m l^{3}}}\end{array}$} & \multicolumn{5}{|c|}{ Displacement ratio $3 d_{\tau} E I / P l^{3}$ at stations-- } & \multicolumn{2}{|c|}{$3 \mathrm{EID} / \mathrm{Pl}^{3}$} & \\
\hline & 1 & 2 & 3 & 4 & 5 & $\mathrm{Eq}(\mathrm{A} 19)$ & $\mathrm{Eq}(\mathrm{A} 20)$ & $\begin{array}{c}\text { ratio at } \\
\text { center, } M / P l\end{array}$ \\
\hline $\begin{array}{r}-3 \\
-2 \\
-1 \\
0 \\
1\end{array}$ & $\begin{array}{r}0 \\
0 \\
0 \\
-0.103900 \\
-.525637\end{array}$ & $\begin{array}{r}0 \\
0 \\
0 \\
-0.049758 \\
-.168315\end{array}$ & $\begin{array}{l}0 \\
0 \\
0 \\
0.049566 \\
.294203\end{array}$ & $\begin{array}{l}0 \\
0 \\
0 \\
0.257936 \\
.967785\end{array}$ & $\begin{array}{l}0 \\
0 \\
0 \\
0.588410 \\
1.83828\end{array}$ & $\begin{array}{l}0 \\
0 \\
0 \\
0.125000 \\
.437499\end{array}$ & $\begin{array}{l}0 \\
0 \\
0 \\
0.125000 \\
.437499\end{array}$ & $\begin{array}{l}0 \\
0 \\
0 \\
.153654 \\
.309800\end{array}$ \\
\hline $\begin{array}{l}2 \\
3 \\
4 \\
5 \\
6\end{array}$ & $\begin{array}{l}-1.40216 \\
-2.72217 \\
-4.42824 \\
-6.50884 \\
-8.99357\end{array}$ & $\begin{array}{r}-.348058 \\
-.596199 \\
-.927607 \\
-1.34718 \\
-1.85109\end{array}$ & $\begin{array}{l}.820339 \\
1.61553 \\
2.64076 \\
3.88834 \\
5.37772\end{array}$ & $\begin{array}{r}\text { 2. } 20772 \\
4.00162 \\
6.35719 \\
9.28142 \\
12.7830\end{array}$ & $\begin{array}{l}\text { 3. } 79212 \\
6.55516 \\
10.2248 \\
14.8320 \\
20.3579\end{array}$ & $\begin{array}{l}.968745 \\
1.73436 \\
2.74216 \\
3.99603 \\
5.49794\end{array}$ & $\begin{array}{l}.968745 \\
1.73436 \\
2.74216 \\
3.99603 \\
5.49794\end{array}$ & $\begin{array}{l}.322636 \\
.253868 \\
.213325 \\
.227965 \\
257022\end{array}$ \\
\hline $\begin{array}{r}7 \\
8 \\
9 \\
10 \\
11\end{array}$ & $\begin{array}{l}-11.9070 \\
-15.2485 \\
-19.0059 \\
-23.1735 \\
-27.7547\end{array}$ & $\begin{array}{l}-2.43581 \\
-3.10178 \\
-3.85121 \\
-4.68514 \\
-5.60308\end{array}$ & $\begin{array}{c}7.12514 \\
9.13013 \\
11.3845 \\
13.8843 \\
16.6319\end{array}$ & $\begin{array}{l}16.8678 \\
21.5368 \\
26.7885 \\
32.6223 \\
39.0387\end{array}$ & $\begin{array}{l}26.7835 \\
34.1121 \\
42.3569 \\
51.5247 \\
61.6123\end{array}$ & $\begin{array}{l}7.24885 \\
9.24923 \\
11.4993 \\
13.9993 \\
16.7491\end{array}$ & $\begin{array}{l}\text { 7. } 24885 \\
9.24923 \\
11.4993 \\
13.9993 \\
16.7491\end{array}$ & $\begin{array}{l}.265110 \\
.254424 \\
.244509 \\
.244722 \\
.249966\end{array}$ \\
\hline $\begin{array}{l}12 \\
13 \\
14 \\
15 \\
16\end{array}$ & $\begin{array}{l}-32.7545 \\
-38.1739 \\
-44.0108 \\
-50.2639 \\
-56.9335\end{array}$ & $\begin{array}{l}-6.60427 \\
-7.68860 \\
-8.85645 \\
-10.1081 \\
-11.4436\end{array}$ & $\begin{array}{l}\text { 19. } 6304 \\
22.8806 \\
26.3810 \\
30.1306 \\
34.1295\end{array}$ & $\begin{array}{l}46.0388 \\
53.6226 \\
61.7899 \\
70.5404 \\
79.8742\end{array}$ & $\begin{array}{r}72.6149 \\
84.5319 \\
97.3652 \\
111.117 \\
125.786\end{array}$ & $\begin{array}{l}\text { 19. } 7488 \\
22.9984 \\
26.4979 \\
30.2473 \\
34.2465\end{array}$ & $\begin{array}{l}19.7488 \\
22.9984 \\
26.4979 \\
30.2473 \\
34.2465\end{array}$ & $\begin{array}{l}.252659 \\
.251377 \\
.249285 \\
.248816 \\
.249636\end{array}$ \\
\hline $\begin{array}{l}17 \\
18 \\
19 \\
20\end{array}$ & $\begin{array}{l}-64.0204 \\
-71.5252 \\
-79.4478 \\
-87.7877\end{array}$ & $\begin{array}{l}-12.8628 \\
-14.3657 \\
-15.9524 \\
-17.6230\end{array}$ & $\begin{array}{l}38.3783 \\
42.8773 \\
47.6262 \\
52.6249\end{array}$ & $\begin{array}{l}89.7915 \\
100.292 \\
111.377 \\
123.044\end{array}$ & $\begin{array}{l}141.372 \\
157.874 \\
175.294 \\
193.631\end{array}$ & $\begin{array}{l}38.4957 \\
42.9946 \\
47.7434 \\
52.7420\end{array}$ & $\begin{array}{l}38.4957 \\
42.9946 \\
47.7434 \\
52.7420\end{array}$ & $\begin{array}{l}250307 \\
.250228 \\
.249824 \\
249634\end{array}$ \\
\hline
\end{tabular}

TABLE 6. Values of $J$ at stations on beam

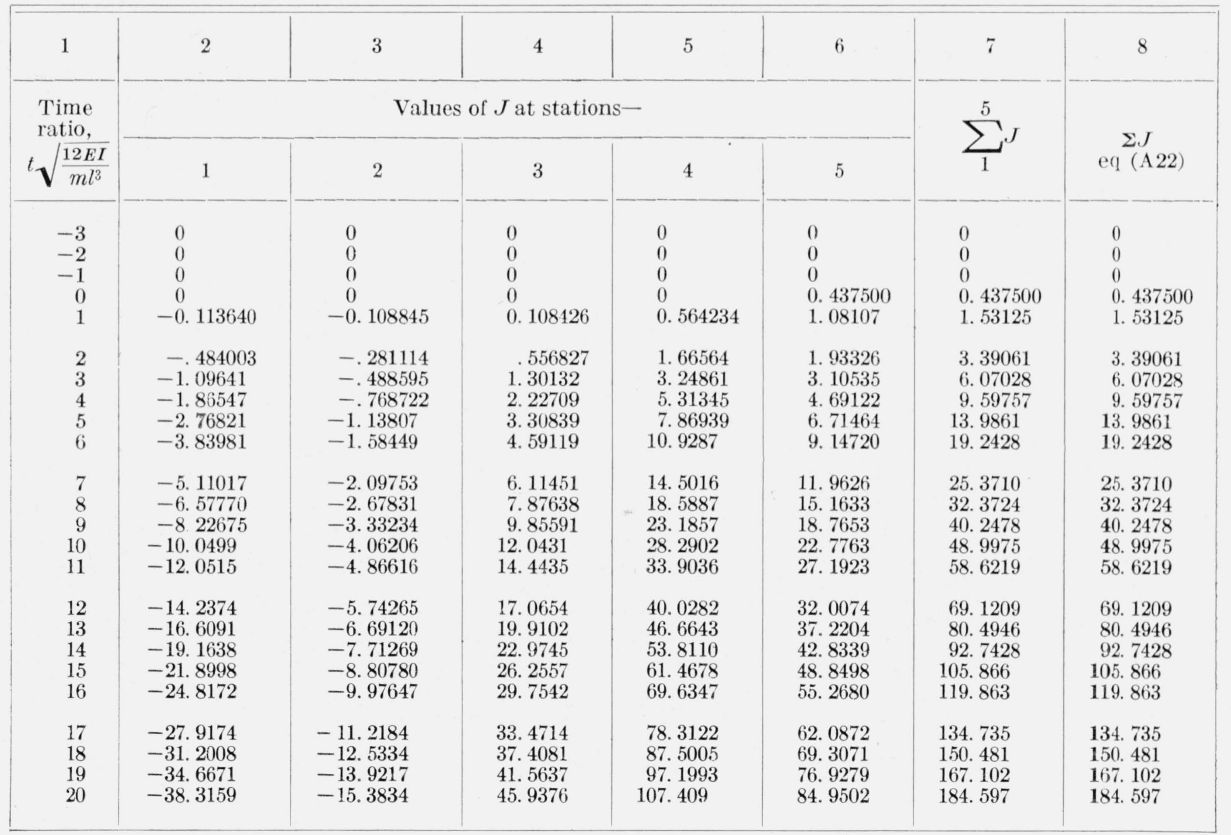


The forces are determined from the displacements of the masses at the five stations on the beam by use of eq (A7). The forces, multiplied by thier distances from the center of the beam determine the bending moment at the center of the beam. Designating the bending moment at the center by $M$,

$$
M=F_{1} l+F_{2} \frac{l}{2}
$$

$$
\begin{array}{r}
M=\frac{48 E I}{7 l^{2}}\left(-0.25 d_{1}+1.50 d_{2}-2.50 d_{3}+\right. \\
\left.1.50 d_{4}-0.25 d_{5}\right)
\end{array}
$$

$$
\begin{array}{r}
\frac{M}{P l}=-0.5714286 \frac{3 d_{1} E I}{P l^{3}}+3.4285714 \frac{3 d_{2} E I}{P l^{3}}- \\
5.7142857 \frac{3 d_{3} E I}{P l^{3}}+3.4285714 \frac{3 d_{4} E I}{P l^{3}}- \\
0.5714286 \frac{3 d_{5} E I}{P l^{3}} .
\end{array}
$$

Values of the bending moment ratio computed from eq (A25) are given in column 9, table 5. They are plotted in figure 12 for $\Delta t=\sqrt{m l^{3} / 12 E I}$.

The authors thank Mrs. L. W. Roberson for computing the responses of the uniform beams to impact loads.

\section{References}

[1] M. A. Biot and R. L. Bisplinghoff, Dynamic loads on airplane structures during landing, NACA ARR 4H10 and Wartime Report W-92 (October 1944).

[2] Walter Ramberg and Albert E. McPherson, Experimental verification of theory of landing impact, NBS J. Research 41, (1948) RP1936.

[3] Walter Ramberg, Transient vibration in an airplane wing obtained by several methods, NBS J. Research 42, 437 (1949) RP1984.

[4] John C. Houbolt, A recurrence matrix solution for the dynamic response of aircraft in gusts, NACA TN 2060 (March 1950).

[5] Wilhelmina D. Kroll and Samuel Levy, A step-by-step method of determining the dynamic response of aircraft in landing, NBS Lab. No. 6.4/1-181, PR6, BuAer TED NBS 2410 (September 21, 1949).

[6] George G. O'Brien, Morton A. Hyman, and Sidney Kaplan, A study of the numerical solution of partial differential equations, Naval Ord. Lab. Memo 10433 (January 4, 1949).

[7] R. Courant, K. Friedricks, and H. Lewy, Über die partiellen Differenzengleichungen der Mathematishen Physik, Math. Annalen, p. 32-74 (1928).

[8] Samuel Levy, Computation of influence coefficients for aircraft structures with discontinuities and sweepback, J. Aeronaut. Sci. [10] 14, 547-560 (October 1947).

[9] W. J. Duncan, Preliminary note on the dependence of the errors in the natural frequencies on the number of elements in the segmental representation of a uniform shaft (April 1950).

Washington, May 3, 1951. 